\title{
An Improved EMD and Its Applications to Find the Basis Functions of EMI Signals
}

\author{
Hongyi Li, Chaojie Wang, and Di Zhao \\ LMIB, School of Mathematics and System Science, Beihang University, Beijing 100191, China \\ Correspondence should be addressed to Di Zhao; zdhyl2010@163.com
}

Received 18 July 2015; Revised 23 October 2015; Accepted 3 November 2015

Academic Editor: Kalyana C. Veluvolu

Copyright ( 2015 Hongyi Li et al. This is an open access article distributed under the Creative Commons Attribution License, which permits unrestricted use, distribution, and reproduction in any medium, provided the original work is properly cited.

\begin{abstract}
A B-spline empirical mode decomposition (BEMD) method is proposed to improve the celebrated empirical mode decomposition (EMD) method. The improvement of BEMD on EMD mainly concentrates on the sifting process. First, instead of the curve that resulted from computing the average of upper and lower envelopes, the curve interpolated by the midpoints of local maximal and minimal points is used as the mean curve, which can reduce the cost of computation. Second, the cubic spline interpolation is replaced with cubic B-spline interpolation on account of the advantages of B-spline over polynomial spline. The effectiveness of BEMD compared with EMD is validated by numerical simulations and an application to find the basis functions of EMI signals.
\end{abstract}

\section{Introduction}

The rapid progress of electronic equipment leads to more and more serious electromagnetic interference (EMI) problems, which will reduce the efficiency of devices. Thus great attentions have been paid to the design of electromagnetic compatibility (EMC) in order to eliminate EMI [1-3]. EMI usually results from many sources but only the mixed signal is known. In order to obtain the useful signal, the EMI noises should be subtracted from the mixed signal. However, the component signals from different sources cannot be distinguished directly and easily. Fortunately, the EMI noises and the useful signals often fall in different range of frequencies. Based on this, the EMI noise can be determined and reduced after the mixed signal is decomposed into basis functions of different frequencies.

Traditional signal decomposition methods like Fourier transform (FT) and wavelet transform (WT) are frequently used to process signals, but they cannot be used to decompose EMI signals effectively since the majority of EMI signals are nonlinear and nonstationary. As is well known, there are certain crucial restrictions on the use of FT and WT. FT is strictly limited to linear and stationary signals. Otherwise, the resulting Fourier spectrum will have little physical significance. WT is basically appropriate for linear signals and is not an adaptive method since the elementary wavelet function has to be selected beforehand, which cannot match the varying nature of the original signals $[4,5]$.

To overcome the disadvantages of FT and WT, Huang et al. have proposed the empirical mode decomposition (EMD) method [6]. By now, EMD has been applied successfully to many fields such as machinery, medicine, and oceanics [79]. EMD is an adaptive method because it can decompose the signal into some intrinsic mode functions (IMFs) that are determined by the signal itself. One of the most important steps of EMD is to determine the mean curve by computing the average of upper and lower envelopes, which are the cubic spline interpolations of maxima and minima points, respectively. But it needs a great deal of calculations and will cause mode mixing problems and overshoot and undershoot problems sometimes. In [10, 11], Chen et al. investigate alternative decomposition algorithms using moving mean averages of the extrema as combinations of B-splines. According to their idea, the mean curve does not pass through the local midpoints, which will lead to bigger local errors. In fact, their goal is merely to establish a firmer mathematical foundation for EMD. Recently, Wang and Li. have proposed the extreme-point symmetric mode decomposition (ESMD) method [12]. Rather than constructing two outer envelopes, the sifting process of ESMD is implemented by the aid of 
some inner curves interpolated by the midpoints of the line segments connecting the local maxima and minima points. Thus ESMD costs less than EMD.

In order to improve these decomposition methods, we present a B-spline empirical mode decomposition (BEMD) method in this paper by replacing the cubic spline interpolation in ESMD with the cubic B-spline interpolation. Therefore, BEMD will not only be more adaptive but also cost less than EMD. The rest of this paper is organized as follows. In Section 2, we provide a brief description of the EMD method. Section 3 introduces the basic theory of B-spline interpolation and discusses its advantages over polynomial spline interpolation. In Section 4, the BEMD method is proposed and analyzed. Section 5 provides the experimental results to demonstrate the validity of BEMD compared with EMD and ESMD. Finally, some conclusions are drawn in Section 6.

\section{The EMD Method}

The essence of EMD is to identify the IMFs through a series of sifting processes empirically. IMFs are supposed to satisfy two conditions: the difference between the number of the extreme points and that of zero crossing points must less than one; the local mean value of the upper and lower envelope at any point should be zero. For a given discrete signal $s(t)$, the main procedures of EMD are as the following [6]:

(i) Connect all the local maxima and minima points of the signal by cubic spline interpolation to construct the upper envelope and lower envelope, respectively.

(ii) Calculate the mean curve $m_{11}(t)$ of two envelopes and extract the difference between $s(t)$ and $m_{11}(t)$ as

$$
h_{11}(t)=s(t)-m_{11}(t) .
$$

Ideally, $h_{11}(t)$ will be the first IMF. Otherwise, take it as a new signal and repeat the above steps:

$$
\begin{aligned}
& h_{12}(t)=h_{11}(t)-m_{12}(t), \\
& h_{13}(t)=h_{12}(t)-m_{13}(t),
\end{aligned}
$$

until it satisfies the two conditions, which are commonly represented by controlling the standard deviation (SD) to lie in $[0.2,0.3]$ :

$$
\mathrm{SD}=\sum_{t=0}^{T}\left(\frac{\left|h_{1(k-1)}(t)-h_{1 k}(t)\right|^{2}}{\left(h_{1(k-1)}(t)\right)^{2}}\right) .
$$

Then the first IMF is obtained as $c_{1}(t)=h_{1 k}(t)$.

(iii) Let the residual $r_{1}(t)=s(t)-c_{1}(t)$ be a new signal. Repeat the above process to obtain the other IMFs $c_{i}(t)$ and the residuals

$$
\begin{aligned}
& r_{2}(t)=r_{1}(t)-c_{2}(t), \\
& r_{3}(t)=r_{2}(t)-c_{3}(t),
\end{aligned}
$$

until the residual $r_{n}(t)$ turns into a monotonic function. Finally, the original signal $s(t)$ can be expressed as

$$
s(t)=\sum_{i=1}^{N} c_{i}(t)+r_{n}(t) .
$$

Using EMD, the original signal can be decomposed into a set of data-driven basis functions, which is actually the monocomponent signals. ESMD is similar to EMD, but the mean curve in its sifting process is constructed by connecting the midpoints of local extrema using cubic spline interpolation. So ESMD is more adaptive and economic than EMD.

\section{The B-Spline Interpolation}

For given increasing sequences $\left\{\tau_{j}, j \in Z\right\}$, the $j$ th B-spline of order $k$ is defined by the $k$ th order divided difference:

$$
B_{j, k, \tau}(t):=\left(\tau_{j+k}-\tau_{j}\right)\left[\tau_{j}, \ldots, \tau_{j+k}\right](\cdot-t)_{+}^{k-1}, \quad t \in R .
$$

These B-splines form a basis for the space of splines of order $k$ with knots $\left\{\tau_{j}, j \in Z\right\}$. Given a set of $n+1$ data points $D_{0}, D_{1}, \ldots, D_{n}$, the $\mathrm{B}$-spline interpolation results in the following approximate curve [13]:

$$
y(x)=\sum_{j=0}^{n} M_{j, k, \tau} B_{j, k, \tau}(x),
$$

where $M_{j, k, \tau}, j=0,1, \ldots, n$ are $n+1$ unknown control points. When the order of the B-spline is $k=3$, they can be obtained quickly similar to those of the cubic spline interpolation by solving a symmetric tridiagonal Toeplitz linear equations using the chasing method:

$$
T x=b,
$$

where

$$
T=\left(\begin{array}{cccc}
4 & 1 & & \\
1 & 4 & \ddots & \\
& \ddots & \ddots & 1 \\
& & 1 & 4
\end{array}\right)
$$

and the right-hand vector $b$ can be computed directly by the given data points according to different kinds of boundary conditions.

It is well known that B-spline, or basis spline, is a spline function that has minimal support with respect to a given degree, smoothness, and domain partition [13]. As it possesses better local properties than the polynomial spline, we replace the cubic spline interpolation with cubic B-spline interpolation for establishing the BEMD method.

As for the simulation signal

$$
s(t)=s_{1}(t)+s_{2}(t)+s_{3}(t), \quad t \in[-\pi, \pi],
$$

where

$$
\begin{aligned}
& s_{1}(t)=3 \cos \left(5 \pi t+\frac{\pi}{2}\right) ; \\
& s_{2}(t)=2 \cos (4 \pi t) ; \\
& s_{3}(t)=\sin (2 \pi t),
\end{aligned}
$$




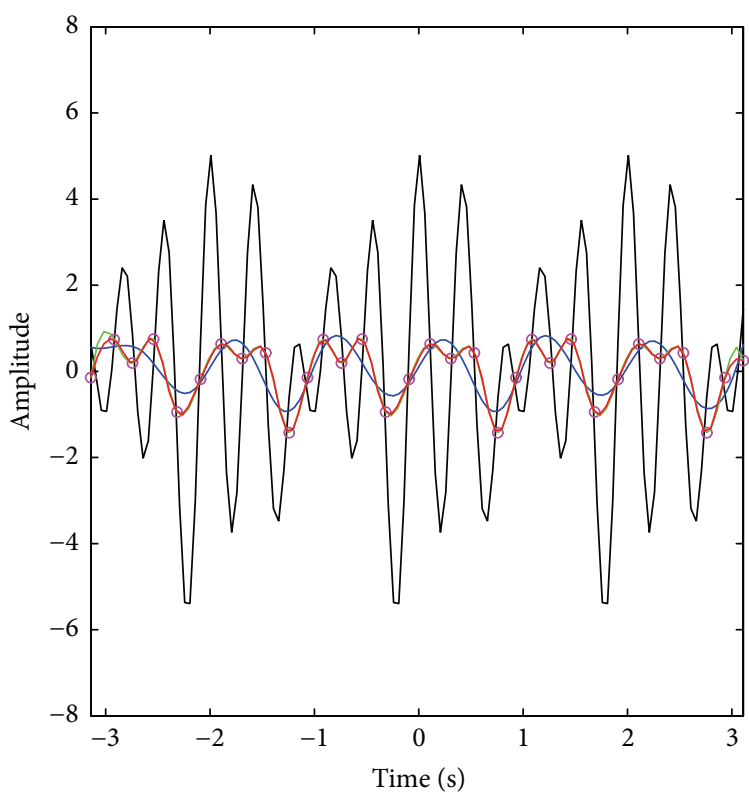

The original signal
The midpoints of local extrema $\quad$ The mean curve of ESMD
The mean curve of EMD

Figure 1: Comparisons between the mean curves of EMD, ESMD, and BEMD.

different methods are used to construct its mean curves and the result is exhibited in Figure 1. It can be seen that differing from those of ESMD and BEMD, the mean curve of EMD constructed by computing the average of the upper and lower envelopes does not pass through the midpoints of local extrema, which will produce bigger local errors. The mean curve of BEMD almost overlaps that of ESMD. However, it is more adaptive because of the better local property of B-spline.

\section{The BEMD Method}

On purpose of improving EMD and ESMD, we present a Bspline empirical mode decomposition (BEMD) method in this section by using the cubic B-spline interpolation instead of the cubic spline interpolation to construct the mean curve. For a given discrete signal $s(t)$, the main procedures of BEMD are as follows:

(i) Find all the local extreme points of $s(t)$; then compute the midpoints of all the adjacent maximal and minimal points.

(ii) Construct the mean curve $m_{11}(t)$ by the cubic Bspline interpolation of theses midpoints.

(iii) Extract the difference between $s(t)$ and $m_{11}(t)$ as (1) and calculate the standard deviation (SD) as (3). If it lies in $[0.2,0.3], h_{11}(t)$ is the first IMF. Otherwise, take $h_{11}(t)$ as a new signal and repeat the above steps as in (2) until SD falls in $[0.2,0.3]$. Then the first IMF is obtained as $c_{1}(t)=h_{1 k}(t)$.
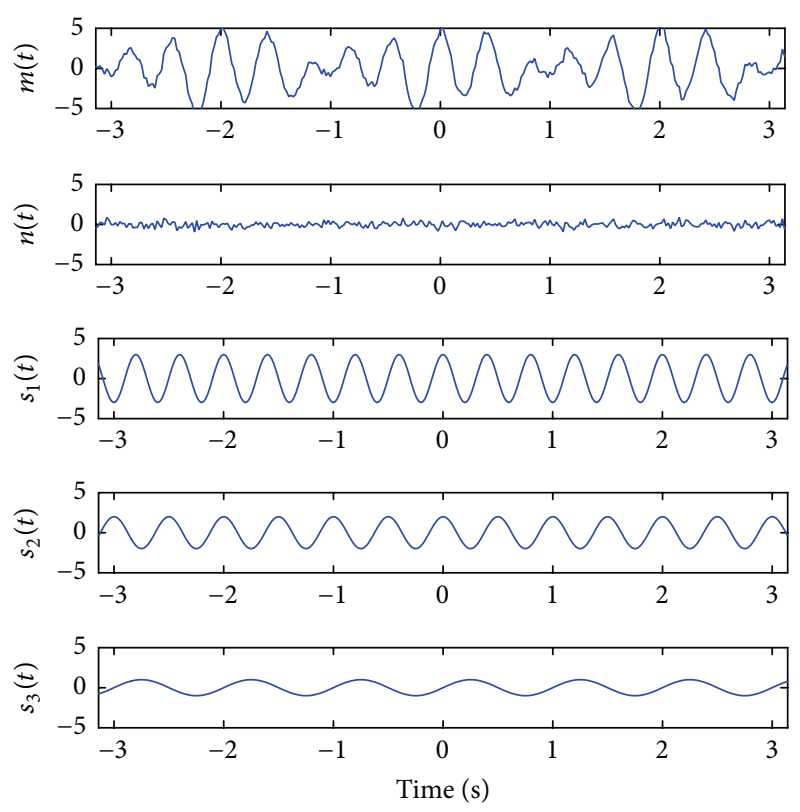

FIGURE 2: The simulation signal $m(t)$ and its component signals.

(iv) Let the residual $r_{1}(t)=s(t)-c_{1}(t)$ be a new signal. Repeat the above process to obtain the other IMFs and residuals as in (4) until the residual $r_{n}(t)$ turns into a monotonic function. Finally, the original signal $s(t)$ can be expressed as (5).

As is seen from the above, only one interpolation is needed in every sifting process of ESMD and BEMD. Moreover, the cost of the cubic B-spline interpolation in BEMD is the same as that of the cubic spline interpolation in EMD and ESMD because they all can be implemented mainly by solving the same kind of linear equations in (8). Thus the computational complexities of generating the mean curves in BEMD and ESMD are both the half of that of EMD. Although ESMD and BEMD require almost the same cost of computation, BEMD is more adaptive than ESMD since the cubic B-spline interpolation rather than the cubic spline interpolation is used in BEMD.

\section{Simulation Evaluations and Applications}

5.1. Applications to Simulation Signals. In order to demonstrate the validity of BEMD, we compare it with EMD and ESMD by decomposing the simulation signal $m(t)$, which is formed by adding a white Gaussian noise to a useful signal with a signal-to-noise ratio (SNR) of $10 \mathrm{db}: m(t)=s(t)+n(t)$, where $s(t)$ is given in (10) with a sample rate of $25 \mathrm{~Hz}$ and $n(t)$ is a white Gaussian noise. The mixed signal $m(t)$ and its components are shown in Figure 2. As is well known, the white Gaussian noise is nonlinear and nonstationary, so $m(t)$ is also nonlinear and nonstationary. As for $m(t)$, the IMFs that resulted from EMD, ESMD, and BEMD are shown in Figures 3(a)-3(c), respectively. Moreover, the component signals of the original signal $s(t)$ and the white Gaussian 

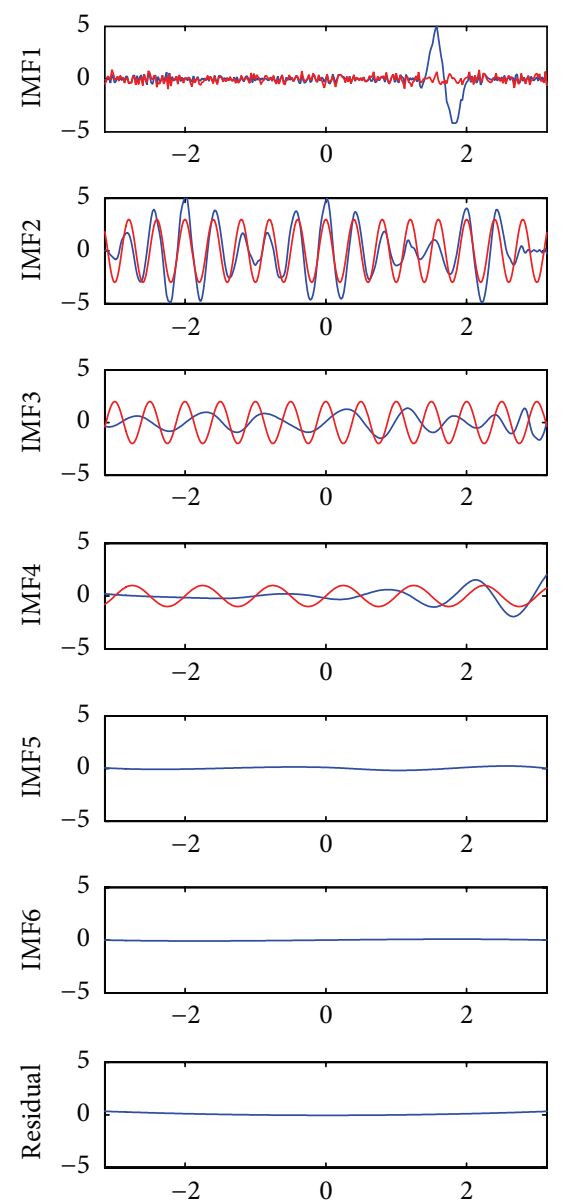

(a)
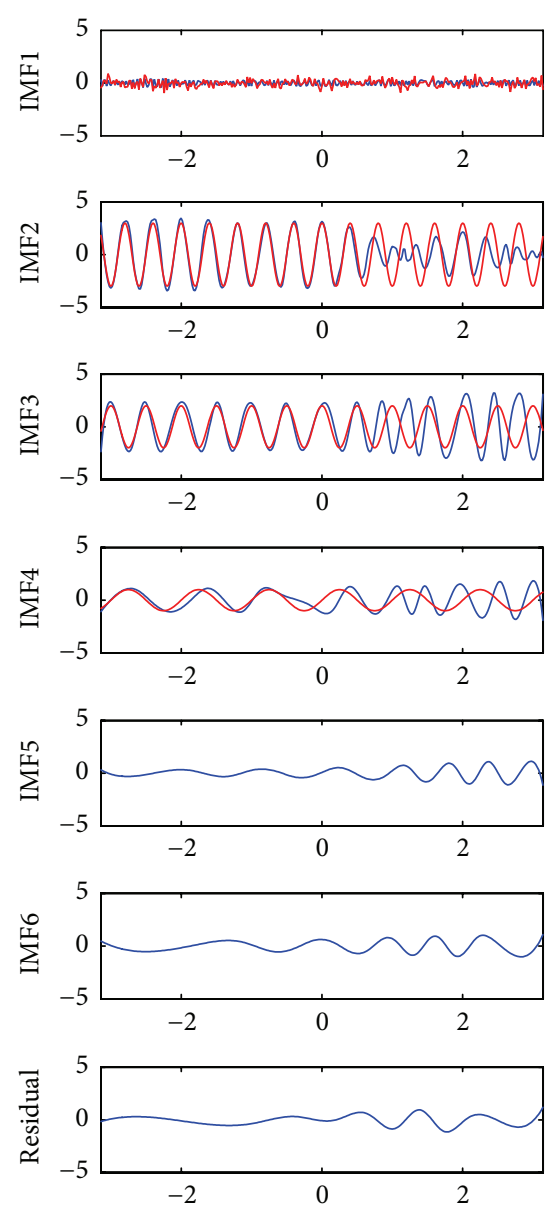

(b)
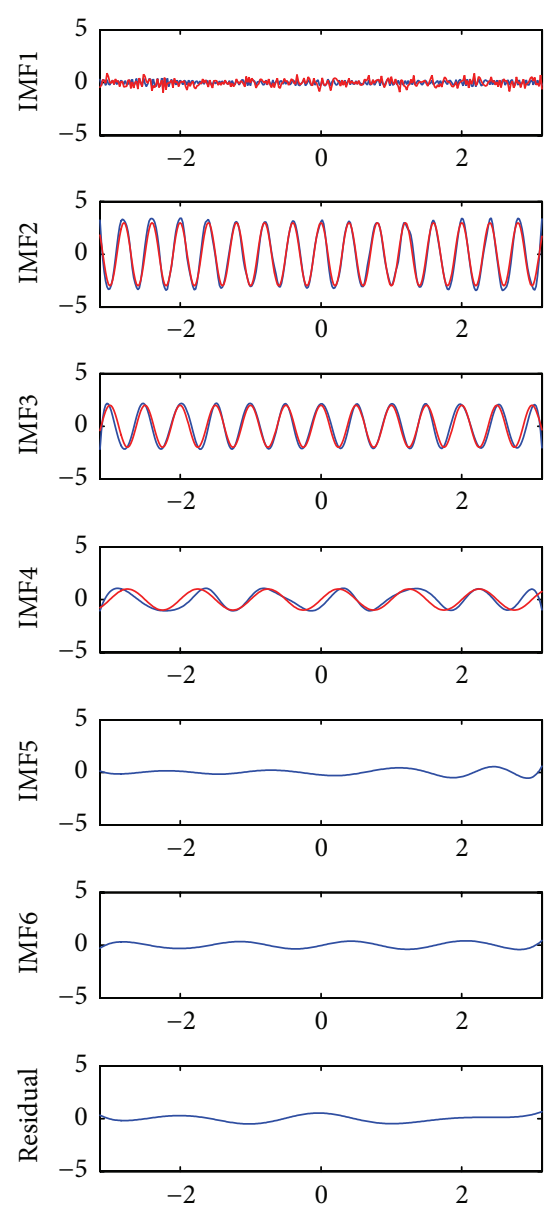

(c)

Figure 3: (a) Decomposition results of $m(t)$ by EMD. (b) Decomposition results of $m(t)$ by ESMD. (c) Decomposition results of $m(t)$ by BEMD.

noise are exhibited along with their corresponding IMFs for comparison.

In Figure 3(a), the IMF1 that resulted from EMD is close to the white Gaussian noise and IMF2 is close to $s_{1}(t)$ even though there exist some big local errors due to some mode mixing problems, but the IMF3 and IMF4 are awfully different from $s_{2}(t)$ and $s_{3}(t)$ on account of some overshoot and undershoot problems. As is seen from Figure 3(b), the local errors between the component signals of $s(t)$ and IMFs resulted from ESMD decrease obviously. But there still exist some mode mixing problems in its IMF2 and some overshoot and undershoot problems in its IMF3 and IMF4. As for BEMD, Figure 3(c) shows the mode mixing problem and overshoot and undershoot problems almost disappear. Its first four IMFs overlap with the waveforms of the white Gaussian noise and the component signals of $s(t)$ more closely than those of EMD and ESMD, which indicates BEMD is more adaptive than EMD and ESMD.

More precisely, we compute the error norms $\operatorname{Err}_{i}(i=$ $1,2,3,4)$ between IMFs and their corresponding component signals as

$$
\operatorname{Err}_{i}=\left\|\mathrm{IMF}_{i}-s_{i}\right\|_{2} .
$$

TABLE 1: The error norms and residual norms of different methods for $m(t)$.

\begin{tabular}{lccccc}
\hline Methods & Err $_{1}$ & $\operatorname{Err}_{2}$ & $\operatorname{Err}_{3}$ & Err $_{4}$ & $\begin{array}{c}\text { Residual } \\
\text { norms }\end{array}$ \\
\hline EMD & 16.4533 & 26.8690 & 29.2602 & 14.5817 & 2.4377 \\
ESMD & 4.6565 & 22.1711 & 28.3135 & 16.2516 & 8.0051 \\
BEMD & 4.7741 & 7.5115 & 8.3750 & 7.6419 & 5.1614 \\
\hline
\end{tabular}

The smaller the error norm is, the closer the IMF is to the component signal. The error norms and the norms of the residuals are listed in Table 1, where $\operatorname{Err}_{1}, \operatorname{Err}_{2}, \operatorname{Err}_{3}$, and $\operatorname{Err}_{4}$ denote the error norms between the white Gaussian noise $n(t)$ and IMF1, $s_{1}(t)$ and IMF2, $s_{2}(t)$ and IMF3, and $s_{3}(t)$ and IMF4, respectively.

As is shown in Table 1, the error norms of BEMD are all smaller than those of EMD and ESMD, which is in accordance with the results provided in Figure 3. Besides, the residual norm of BEMD is bigger than that of EMD and is less than that of ESMD. According to Huang's theory in [11], 

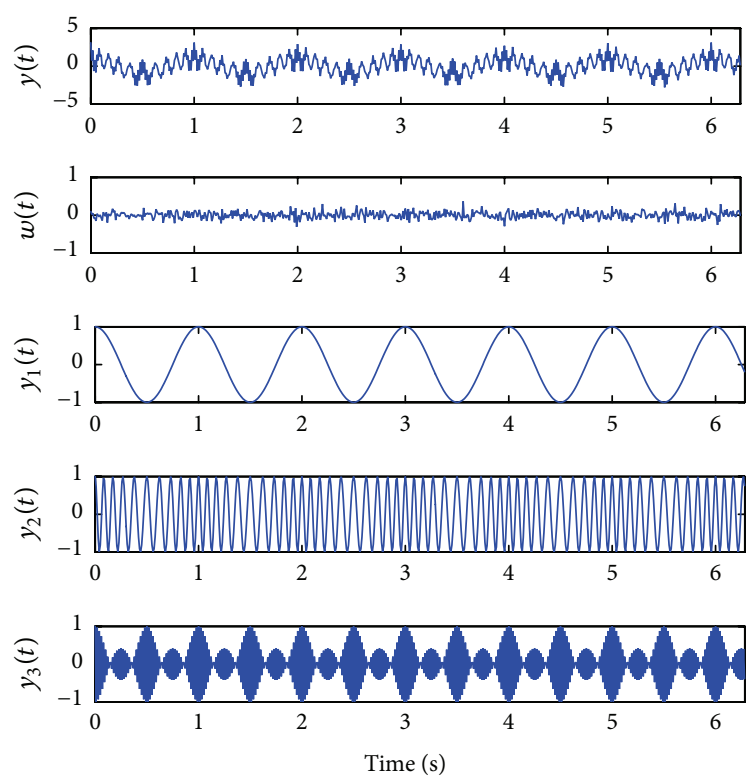

Figure 4: The EMI signal $y(t)$ and its component signals.

the residual can be either the mean trend or a constant. In fact, the evaluation of a EMD-like method depends on not the value of its residual norm but whether the signal can be decomposed into physically meaningful representations. Therefore, the results in Figure 3 and Table 1 both show that BEMD outperforms EMD and ESMD for decomposing the nonlinear and nonstationary simulation signal $m(t)$.

5.2. Applications to EMI Signals. As is well known, there are a large scale of amplitude modulation (AM) and frequency modulation (FM) signals in our surrounding environment such as radio signals, radar signals, and sonar signals [14, 15], which can influence the normal status of equipment. In order to reduce electromagnetic interference by subtracting the noise components from the mixed signal, the basis functions corresponding to the EMI noises should be found first using certain decomposition methods. According to the theory of EMD-like methods, the intrinsic mode functions (IMFs) can be regarded as the basis functions because they are some physically meaningful representations of the signal [11].

As for a harmonic wave $y_{1}(t)=\cos (2 \pi t)$ with a sample rate of $100 \mathrm{~Hz}$, when it is interfered by two FM signals $y_{2}(t)=$ $\cos (20 \pi t+2 \sin (2 t))$ and $y_{3}(t)=\cos (500 \pi t+2 \sin (2 t))$ and a white Gaussian noise $w(t)$ with a SNR of $20 \mathrm{db}$, it forms the following nonlinear and nonstationary EMI signal:

$$
y(t)=y_{1}(t)+y_{2}(t)+y_{3}(t)+w(t), \quad t \in[0,2 \pi],
$$

which is shown in Figure 4. We decompose $y(t)$ by EMD, ESMD, and BEMD and show the results in Figure 5.

As is shown in Figure 5(a), the IMF1 and IMF2 overlap with the component signal $y_{3}(t)$ and $y_{2}(t)$ well. But there exists an obvious mode mixing problem of IMF3 even though it behaves like the harmonic wave $y_{1}(t)$. Besides, the decomposition results do not include a corresponding IMF to
TABLE 2: The error norms of different methods for $y(t)$.

\begin{tabular}{lcccc}
\hline Methods & Err $_{1}$ & Err $_{2}$ & Err $_{3}$ & Err $_{4}$ \\
\hline EMD & - & 9.2599 & 3.8289 & 3.8118 \\
ESMD & - & 3.9242 & - & 2.6267 \\
BEMD & 3.4426 & 7.3495 & 2.0984 & 1.5690 \\
\hline
\end{tabular}

the white Gaussian noise $w(t)$. In Figure 5(b), IMF1 and IMF8 behave as the waveform of $y_{3}(t)$ and $y_{1}(t)$ well. However, there is no IMF that corresponds to $y_{2}(t)$ or the white Gaussian noise $w(t)$. In addition, the IMF2, IMF3, and IMF4 have severe mode mixing problems. As for the decomposition results of $y(t)$ by BEMD in Figure 5(c), its IMF1, IMF2, IMF3, and IMF7 exactly show the waveforms of $y_{3}(t)$, the white Gaussian noise $w(t), y_{2}(t)$, and $y_{1}(t)$, respectively. In a word, BEMD produces more meaningful IMFs than EMD and ESMD and it can alleviate the mode mixing problem.

More accurately, the error norms $\operatorname{Err}_{i}(i=1,2,3,4)$ as in (12) are computed and listed in Table 2, where $\operatorname{Err}_{1}, \operatorname{Err}_{2}, \operatorname{Err}_{3}$, and $\operatorname{Err}_{4}$ denote the error norms between $w(t), y_{1}(t), y_{2}(t)$, $y_{3}(t)$, and their corresponding IMFs, respectively. When the decomposition results cannot give the corresponding meaningful IMF of a component signal of $y(t)$, the error norm will be denoted as “-” in Table 2 .

As is shown in Table 2, the decomposition results of BEMD include more meaningful IMFs than those of EMD and the error norms of BEMD are all less than the existing ones of EMD. As for ESMD, its $\mathrm{Err}_{4}$ is bigger than that of BEMD and its $\operatorname{Err}_{2}$ is less than that of BEMD. However, the decomposition results of BEMD include two more meaningful basis functions than those of ESMD.

As for an AM signal $z_{1}(t)=(1+\cos (\pi t)) \cos (2 \pi t)$ with a sample rate of $100 \mathrm{~Hz}$, when it is interfered by an FM signal $z_{2}(t)=\cos (500 \pi t+2 \sin (2 t))$ and a white Gaussian noise $v(t)$ with a SNR of $20 \mathrm{db}$, it forms the following nonlinear and nonstationary EMI signal:

$$
z(t)=z_{1}(t)+z_{2}(t)+v(t), \quad t \in[0,2 \pi],
$$

which is shown in Figure 6. We decompose the AM-FM signal $z(t)$ by EMD, ESMD, and BEMD and exhibit the results in Figure 7.

As is shown in Figure 7, all the IMF1 and IMF2 behave as the waveforms of $z_{2}(t)$ and $v(t)$ well. The difference of three decomposition results mainly lies in the IMFs corresponding to $z_{1}(t)$. Although the IMF4 in Figure $7(\mathrm{a})$ and the residual in Figure $7(\mathrm{~b})$ are close to $z_{1}(t)$, there exist some obvious local errors. As to the residual in Figure $7(\mathrm{c})$, it overlaps with $z_{1}(t)$ more closely, which indicates that the residual of BEMD represents the trend of $z(t)$ better than those of EMD and ESMD.

More clearly, the error norms $\operatorname{Err}_{i}(i=1,2,3,4)$ as in (12) are computed and listed in Table 3, where $\operatorname{Err}_{1}, \operatorname{Err}_{2}$, and $\operatorname{Err}_{3}$ denote the error norms between $v(t), z_{1}(t), z_{2}(t)$, and their corresponding IMFs, respectively. As is seen from Table 3, all the error norms of BEMD are less than those of EMD 

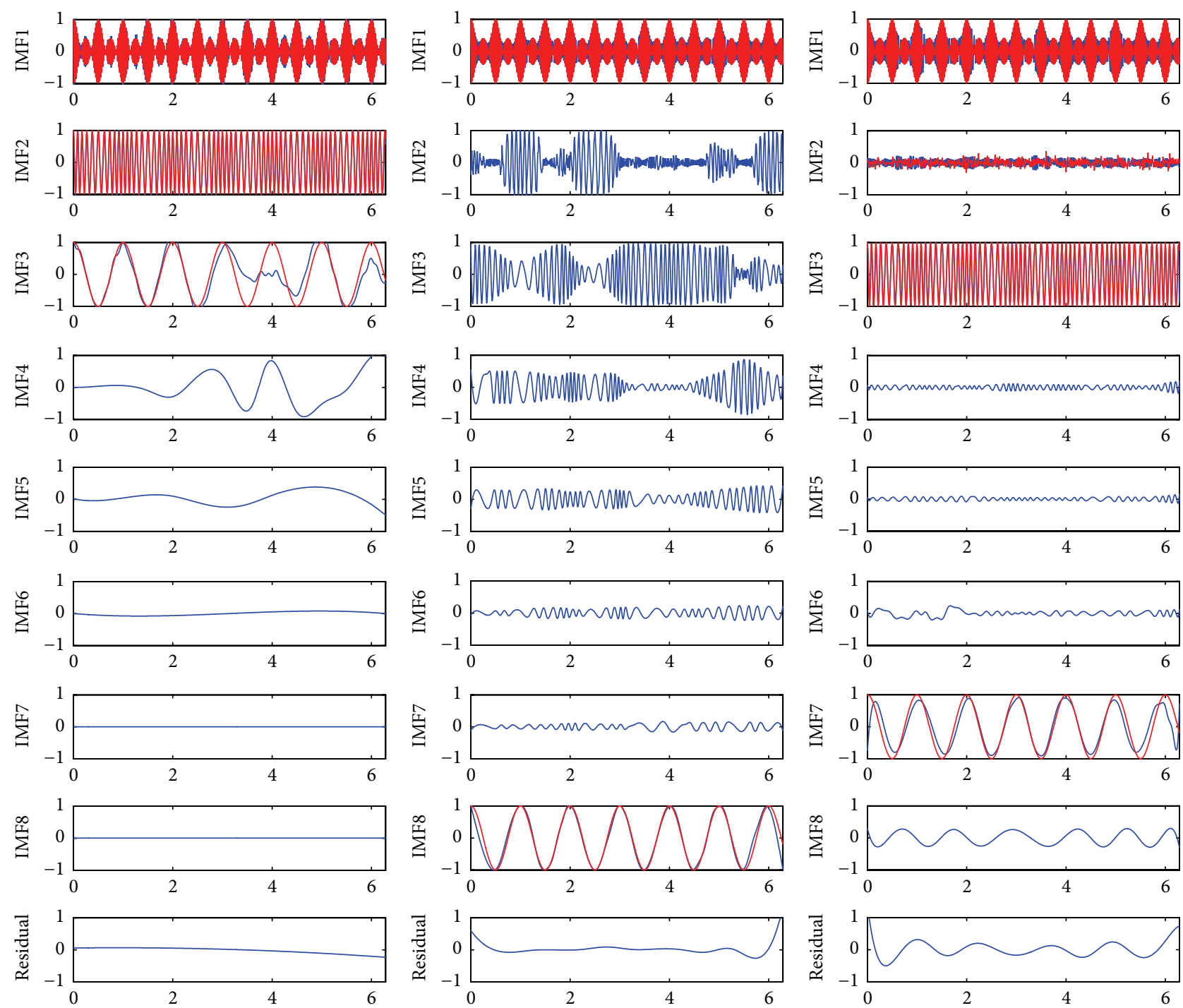

(a)

(b)

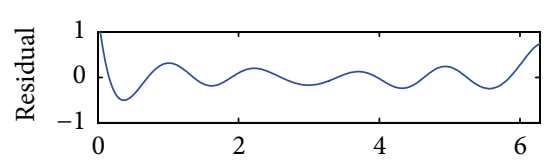

(c)

FIGURE 5: (a) Decomposition results of $y(t)$ by EMD. (b) Decomposition results of $y(t)$ by ESMD. (c) Decomposition results of $y(t)$ by BEMD.

TABLE 3: The error norms of different methods for $z(t)$.

\begin{tabular}{lccc}
\hline Methods & $\mathrm{Err}_{1}$ & $\mathrm{Err}_{2}$ & $\mathrm{Err}_{3}$ \\
\hline EMD & 2.9022 & 15.3004 & 2.5125 \\
ESMD & 3.3348 & 4.3362 & 3.1363 \\
BEMD & 1.9422 & 0.9949 & 1.6007 \\
\hline
\end{tabular}

and ESMD. Therefore, BEMD performs better than EMD and ESMD for finding the basis functions of the nonlinear and nonstationary EMI signals.

\section{Conclusions}

Based on ESMD and the cubic B-spline interpolation, we have presented a new improved form of EMD, which is called BEMD. The mean curve in the sifting process of BEMD is constructed by the cubic B-spline interpolation of the local midpoints, which can not only increase the adaptivity but also decrease the computation cost of EMD. The comparison results of the simulation signal show that BEMD performs better than EMD and ESMD. Moreover, BEMD is verified to be more effective than EMD and ESMD for finding the basis functions of EMI signals.

\section{Conflict of Interests}

The authors declare that there is no conflict of interests regarding the publication of this paper.

\section{Acknowledgment}

The project was supported by the National Natural Science Foundation of China (Grant no. 61379001). 

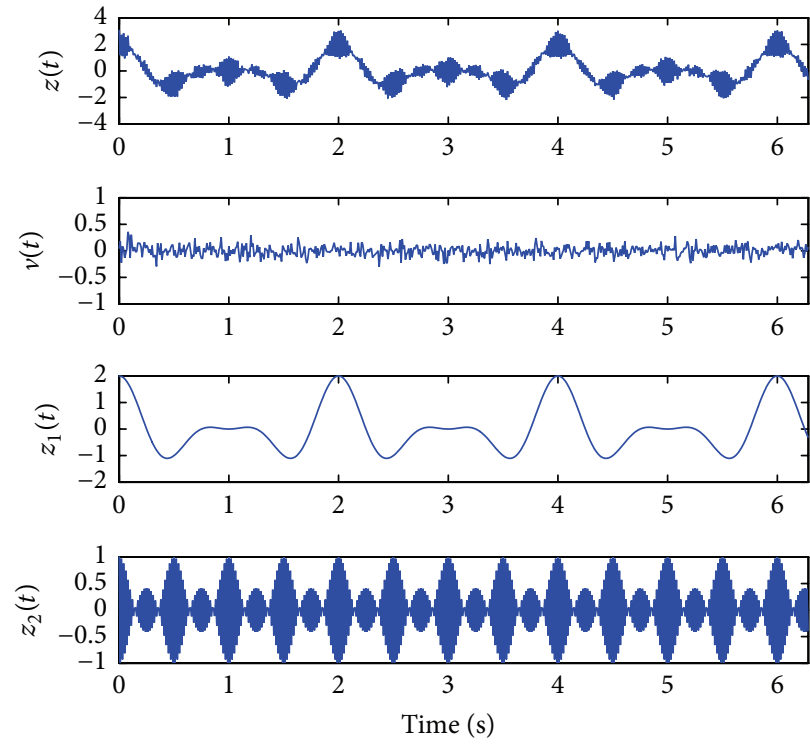

FIGURE 6: The EMI signal $z(t)$ and its component signals.
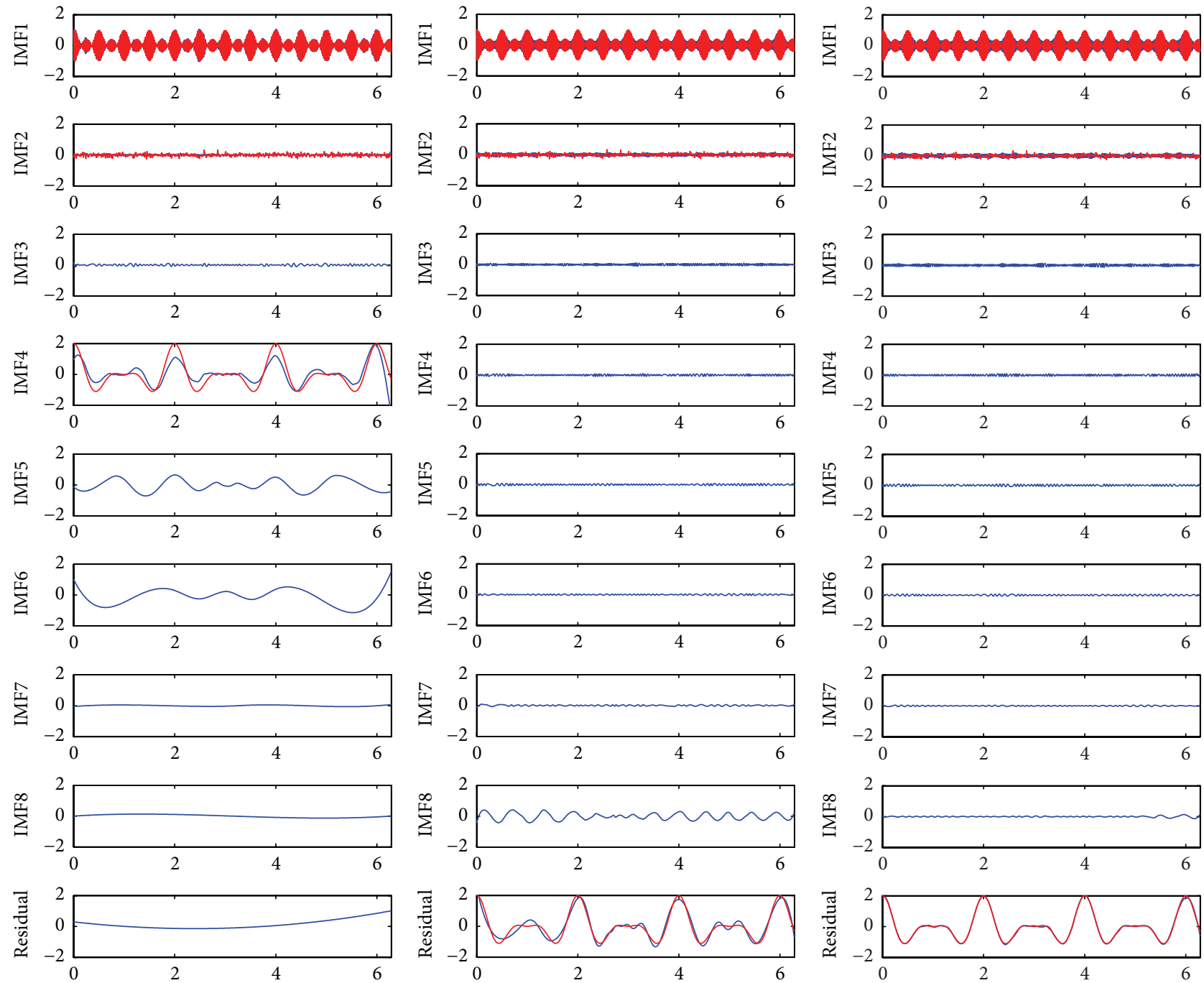

(a)

(b)

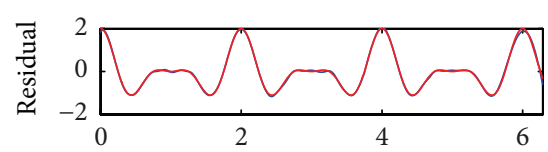

(c)

Figure 7: (a) Decomposition results of $z(t)$ by EMD. (b) Decomposition results of $z(t)$ by ESMD. (c) Decomposition results of $z(t)$ by BEMD. 


\section{References}

[1] H. Guo, H. Wu, B. Zhang, and Z. Li, "Research on periodic switching frequency modulation for conducted EMI suppressing in power converter," Microelectronics Journal, vol. 42, no. 2, pp. 415-421, 2011.

[2] L. Kocewiak, I. Arana, J. Hjerrild, T. Sørensena, C. L. Bakb, and L. Holbøllc, "GPS synchronization and EMC of harmonic and transient measurement equipment in offshore wind farms," Energy Procedia, vol. 24, pp. 212-228, 2012.

[3] N. Meslem, V. T. H. Le, C. Labarre, S. Lecoeuche, J.-L. Kotny, and N. Idir, "Set-membership methods applied to identify highfrequency elements of EMI filters," Control Engineering Practice, vol. 29, pp. 13-22, 2014.

[4] H. Ding, Z. Huang, Z. Song, and Y. Yan, "Hilbert-Huang transform based signal analysis for the characterization of gasliquid two-phase flow," Flow Measurement and Instrumentation, vol. 18, no. 1, pp. 37-46, 2007.

[5] C. J. Wang, H. Y. Li, W. Xiang, and D. Zhao, "A new signal classification method based on EEMD and FCM and its application in bearing fault diagnosis," Applied Mechanics and Materials, vol. 602-605, pp. 1803-1806, 2014.

[6] N. E. Huang, Z. Shen, S. R. Long et al., "The empirical mode decomposition and the Hilbert spectrum for nonlinear and non-stationary time series analysis," Proceedings of the Royal Society A: Mathematical, Physical and Engineering Science, vol. 454, pp. 903-995, 1998.

[7] Z. K. Peng, P. W. Tse, and F. L. Chu, "An improved HilbertHuang transform and its application in vibration signal analysis," Journal of Sound and Vibration, vol. 286, no. 1-2, pp. 187205, 2005.

[8] L. Liu, S. Ma, S. Feng, Y. Jiang, J. Ma, and B. Zhang, "Envelope extraction of Anaesthesia breathing sound signal on Hilbert Huang transform," Procedia Engineering, vol. 2, pp. 2693-2698, 2012.

[9] O. Adam, "The use of the Hilbert-Huang transform to analyze transient signals emitted by sperm whales," Applied Acoustics, vol. 67, no. 11-12, pp. 1134-1143, 2006.

[10] Q. Chen, N. Huang, S. Riemenschneider, and Y. Xu, "A Bspline approach for empirical mode decompositions," Advances in Computational Mathematics, vol. 24, no. 1-4, pp. 171-195, 2006.

[11] N. E. Huang and S. Shen, Hilbert-Huang Transform and Its Applications, World Scientific, Singapore, 2005.

[12] J.-L. Wang and Z.-J. Li, "Extreme-point symmetric mode decomposition method for data analysis," Advances in Adaptive Data Analysis, vol. 5, no. 3, pp. 135-170, 2013.

[13] Y. Feng, F. Zeng, and J. Deng, Spline Functions and Approximation Theory, University of Science and Technology of China Press, Hefei, China, 2013.

[14] X. Guanlei, W. Xiaotong, X. Xiaogang, and Z. Lijia, "Improved EMD for the analysis of FM signals," Mechanical Systems and Signal Processing, vol. 33, pp. 181-196, 2012.

[15] A. Bouchikhi and A.-O. Boudraa, "Multicomponent AM-FM signals analysis based on EMD-B-splines ESA," Signal Processing, vol. 92, no. 9, pp. 2214-2228, 2012. 


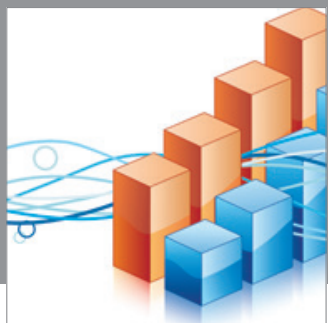

Advances in

Operations Research

mansans

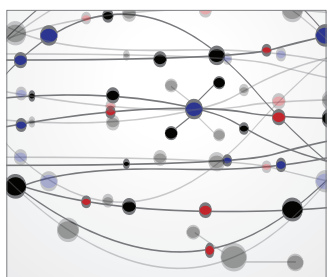

The Scientific World Journal
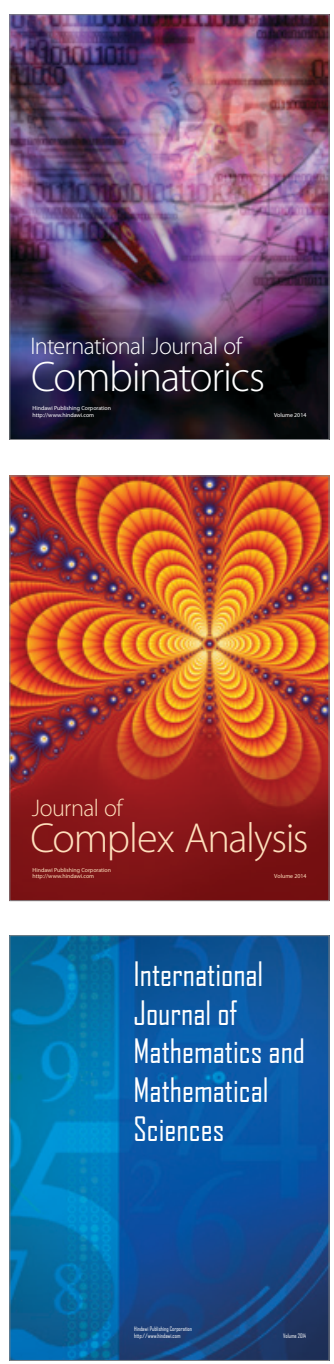
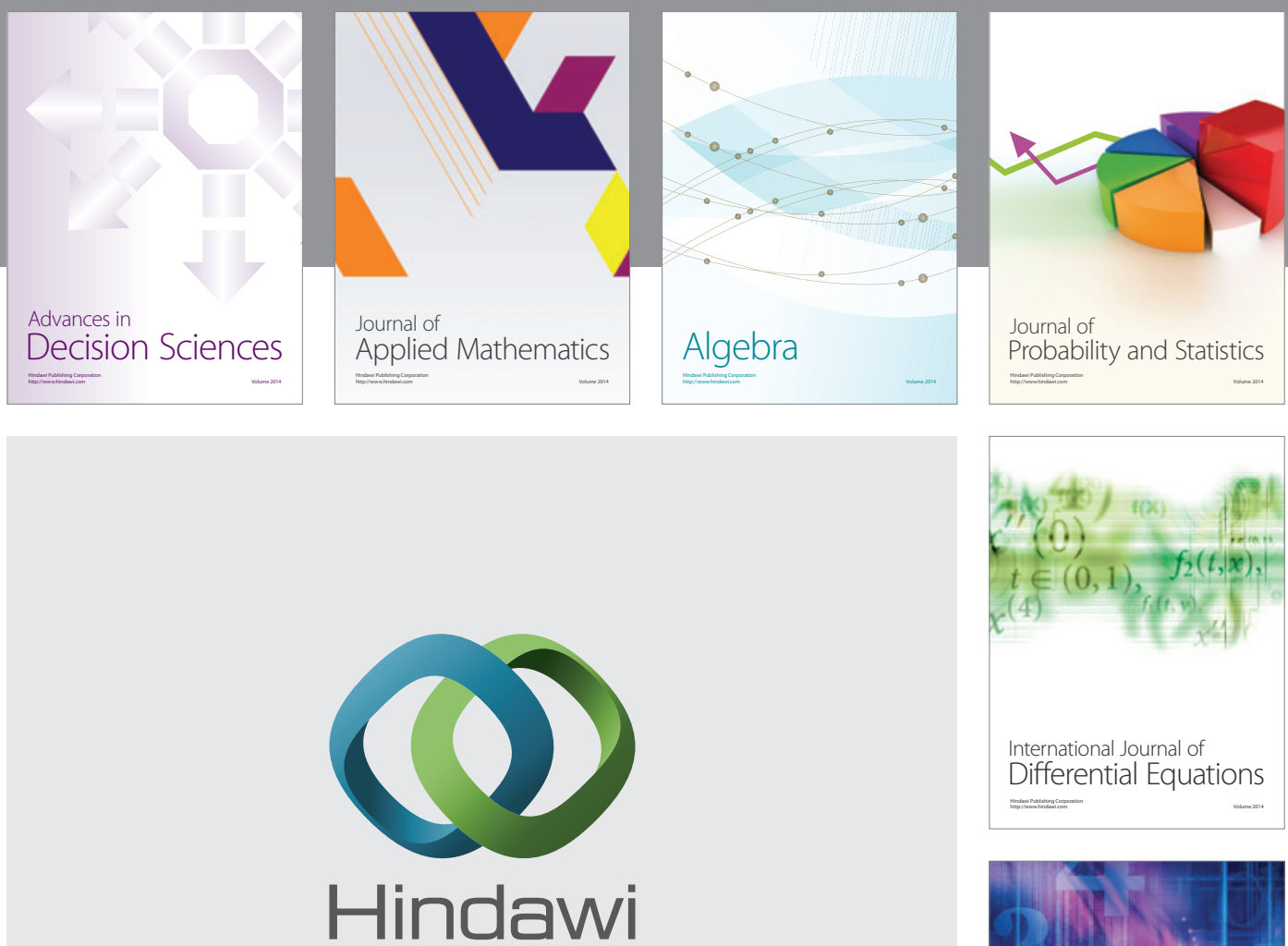

Submit your manuscripts at http://www.hindawi.com
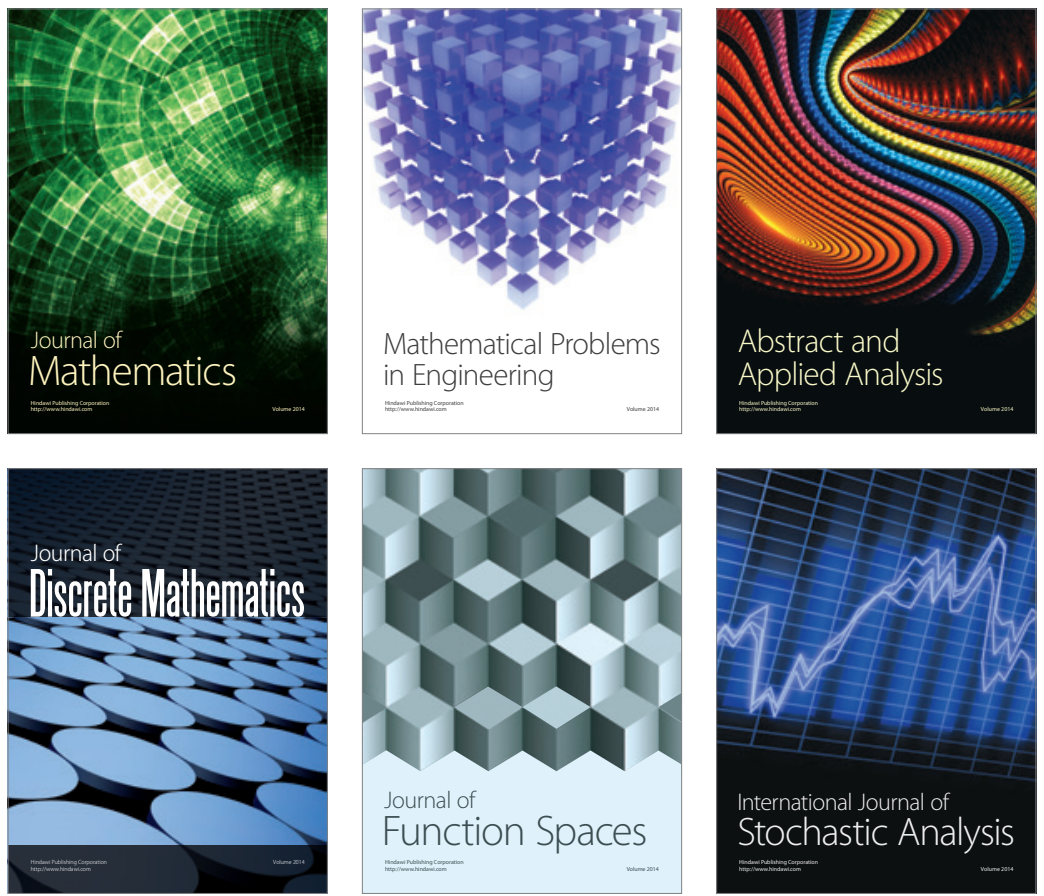

Journal of

Function Spaces

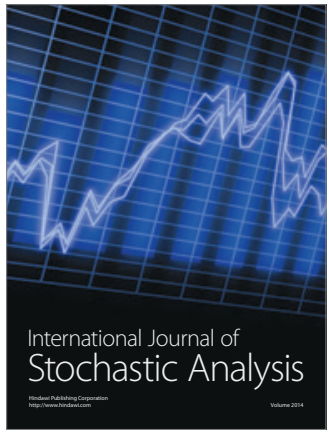

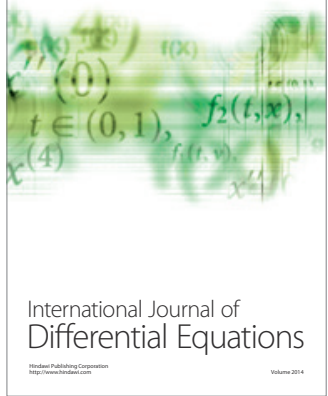
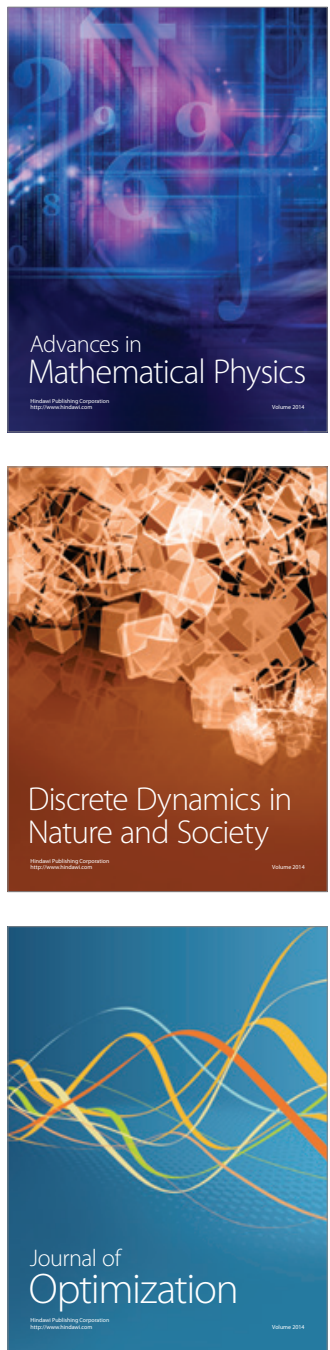